SYNAPTIC TRANSMISSION IN A DIFFUSION MODEL FOR NEURAL ACTIVITY

Vera Lansky, Petr Lansky, Charles E. Smith

Institute of Statistics Mimeo Series No. 2238

Biomathematics Series No. 40 


\section{Synaptic Transmission in a Diffusion Model for Neural Activity}

VĚrA LÁNSKÁ ${ }^{1}$, PETR LÁNSKÝ́ ${ }^{2}$ and CHARLES E. SMITH ${ }^{3}$

1 Department of Statistics, Institute for clinical and Experimental Medicine, Videñská 800, 14000 Prague 4, Czechoslovakia

2 Institute of Physiology, Czechoslovak Academy of Sciences, Videñskí 1083, 14220 Prague 4, Czechoslovakia

3 Biomathematics Program, Department of Statistics, North Carolina State University, Raleigh, North Carolina 27695-8203, U.S.A.

Address for correspondence:

Petr Lánský

Institute of Physiology

Czechoslovak Academy of Sciences

Vídeňská 1083

14220 Prague 4

Czechoslovakia

$\mathrm{FAX}+42-2-4719517$ 
Equations for a diffusion neuronal model describing the production of nerve impulses have been derived for the case in which the noisy depolarizations of the membrane potential are restricted by the reversal potentials. Identifying neuron firing intervals with the firstpassage time distribution for the associated process allows us to to compute the interspike interval statistics and relate them to the parameters characterising the neuronal input. Methods for approximation of the first two moments of the interspike intervals are proposed. The analytical results are numerically illustrated and simultaneously the computer simulations were performed for the same purpose. A comparison of the achieved results is made with those from other diffusion models or the models with discontinuous trajectories. The model is proposed mainly as an alternative to the Ornstein-Uhlenbeck neuronal model.

\section{Introduction}

The Ornstein-Uhlenbeck diffusion process has often been used as a model of the subthreshold membrane potential of a nerve cell. Ricciardi and Sacerdote (1979) studied this model in very detailed way illustrating their results by many numerical examples on the role of parameters in the neuronal inputoutput dynamics. This neuronal model has been usually derived as a diffusion approximation of Stein's model (Stein, 1965) in which the trajectory of the membrane potential is described by a stochastic process with discontinuities (jumps). The main features of Stein's model are linear summation of synaptic inputs and spontaneous exponential decay of the membrane potential. While the diffusion process is easier for the mathematical handling than its discontinuous counterpart, the biological interpretation is more straightforward for the original discontinuous version. The relation between both of them was studied by Tuckwell and Cope (1980).

It is a well know fact that the change of the membrane depolarization by a synaptic input depends on the actual value of the membrane potential. The depolarization of the potential caused by an excitatory postsynaptic potential (EPSP) decreases with decreasing distance of the membrane potential from the excitatory reversal potential, $V_{E}$, which is halfway between the sodium and potassium 
equilibrium potentials (Schmidt, 1984). In the same manner, the hyperpolarization caused by inhibitory postsynaptic potential (IPSP) is smaller if the membrane potential is closer to the inhibitory reversal potential, $V_{I}$. The inhibitory reversal potential is identified with the mean of the equilibrium potential for $\mathrm{K}^{+}$and $\mathrm{Cl}^{-}$, Schmidt (1984).

To reflect nonlinear synaptic summation the existence of the reversal potential was introduced into neural modelling by modifying of the original Stein's model (Tuckwell, 1979). As for the basic model, also for its modification with the reversal potentials, the analysis is complicated and thus the diffusion variants have been examined (Hanson and Tuckwell, 1983; Kallianpur and Wolpert, 1987; Lánský and Lánská, 1987; Giorno et al., 1988). While Stein's model has been always substituted by the Ornstein-Uhlenbeck process, there is a whole class of diffusion processes which can be substituted for the Stein's model with reversal potentials. Which one of the diffusions is used, depends on the fact how the form of Stein's model with reversal potential is modified. In the present paper one of the alternatives is studied.

Here, a stochastic process $X=\{X(t) ; t \geq 0\}$ represents changes in the membrane potential between two consecutive neuronal firings (spikes) and $t$ represents the time since the last spike. The reference level for the membrane potential is taken to be the resting potential. This transformation is formal and starts to play its role only when the parameters of the model are identified. An action potential (spike) is produced when the membrane voltage $X$ exceeds for the first time a voltage threshold, for simplicity assumed to be equal to a constant $S$. The initial voltage (the reset value following a spike) is denoted by $x_{0}$ and is often assumed to be equal to the resting potential, $x_{0}=0$. The interspike interval (ISI) corresponds under this model to the first passage time (FPT) for the associated stochastic process $X$. Thus in studies on neuronal models we are mainly interested in the properties of the random variable $T_{S, x_{0}}$, which is defined by the relationship

$$
T_{S, x_{0}}=\inf \left\{t \geq 0 ; X(t) \geq S \mid X(0)=x_{0}<S\right\}
$$

The assumed resetting mechanism ensures that ISIs form renewal process which is completely described by probability distribution function $G_{S}\left(x \mid x_{0}\right)$, resp. probability density function $g_{S}\left(x \mid x_{0}\right)$, of $T_{S, x_{0}}$. The central moments of $T_{S, x_{0}}$, denoted by $M_{n}\left(S \mid x_{0}\right)(n=1,2, \ldots)$, are often computed in both experimental 
and theoretical studies on neuronal firing. Detailed introduction and reasoning for this type of ISI modeling can be found in Tuckwell (1988).

Before going into the details on the studied model, let us shortly summarize the relevant results from the theory of stochastic diffusion processes. The detailed information can be found in Karlin and Taylor (1981), Ricciardi and Sato (1990). Any time-homogeneous diffusion process (continuous Markov process $X(t)$ satisfying certain regularity conditions) can be characterized by the conditional first and second moments of the change in an infinitesimal time interval:

$$
\begin{gathered}
\mu(x)=\lim _{\Delta t \rightarrow 0_{+}} \frac{E\{X(t+\Delta t)-X(t) \mid X(t)=x\}}{\Delta t} \\
\sigma^{2}(x)=\lim _{\Delta t \rightarrow 0_{+}} \frac{E\left\{[X(t+\Delta t)-X(t)]^{2} \mid X(t)=x\right\}}{\Delta t}
\end{gathered}
$$

We call $\mu(x)$ the drift coefficient (infinitesimal mean) and $\sigma^{2}(x)$ is called the diffusion coefficient (infinitesimal variance). The diffusion process has a transition probability density $f_{x_{0}}(x, t)$, which is the density of $X(t)$, given that $X(0)=x_{0}$. This density satisfies Kolmogorov's forward and backward equations

$$
\begin{aligned}
& \frac{1}{2} \frac{\partial^{2}\left[\sigma^{2}(x) f_{x_{0}}(x, t)\right]}{\partial x^{2}}-\frac{\partial\left[\mu(x) f_{x_{0}}(x, t)\right]}{\partial x}=\frac{\partial f_{x_{0}}(x, t)}{\partial t} \\
& \frac{1}{2} \sigma^{2}\left(x_{0}\right) \frac{\partial^{2} f_{x_{0}}(x, t)}{\partial x_{0}^{2}}+\mu\left(x_{0}\right) \frac{\partial f_{x_{0}}(x, t)}{\partial x_{0}}=\frac{\partial f_{x_{0}}(x, t)}{\partial t}
\end{aligned}
$$

with initial conditions $f_{x_{0}}(x, 0)=\delta\left(x_{0}-x\right)$ (the Dirac $\delta$-function). The integration problem of the diffusion equations (1.4) and (1.5) is complicated by the circumstance that $\sigma^{2}(x)$ may become singular as $x$ approaches some value $x^{*}$, that is called a singular point. When the process has such a singular point, then its behaviour at this point has to be determined and additional boundary condition specified.

If there exists a steady state distribution, $f(x)$, describing the diffusion process after an infinitely long time interval, then $f(x)=f_{x_{0}}(x,+\infty)$. The steady state distribution can be directly computed without the knowledge of the transition density from the formula 


$$
f(x)=\frac{c}{\sigma^{2}(x)} \exp \left(\int^{x} \frac{2 \mu(y)}{\sigma^{2}(y)} \mathrm{d} y\right)
$$

where $c$ is a constant ensuring $f(x)$ to be the probability density function over the state space of the diffusion.

For the reason of simplicity, it is sometimes useful to transform the original process $X$, whose state space is an interval having endpoints $l_{1}$ and $l_{2}$, on a different interval. A continuous strictly monotone function $k$ with two uniformly continuous derivatives $k$ ' and $k$ " may be used and it transforms the process $X$ with infinitesimal moments (1.2) and (1.3) into a new process $Y(t)=k(X(t))$ defined on the interval with endpoints $k\left(l_{1}\right)$ and $k\left(l_{2}\right)$ and having infinitesimal moments

$$
\begin{gathered}
\mu_{Y}(y)=\frac{1}{2} \sigma^{2}(x) k^{\prime}(x)+\mu(x) k^{\prime}(x) \\
\sigma_{Y}^{2}(y)=\sigma^{2}(x)\left\{k^{\prime}(x)\right\}^{2}
\end{gathered}
$$

where $y=k(x)$.

The FPT density function $g_{S}\left(x \mid x_{0}\right)$ can be derived from the transition density function using Fortet's equation connecting these two densities,

$$
f_{x_{0}}(x, t)=\int_{0}^{t} g_{S}\left(\tau \mid x_{0}\right) f_{S}(x, t-\tau) \mathrm{d} \tau
$$

holding for $x \geq S$. This integral equation, putting $x=S$, has been a starting point for many numerical calculations of FPT densities, namely when a time dependent thresholds for Wiener process have been considered, (Durbin, 1971). The method of Durbin was extended to the case of temporally inhomogeneous diffusion and applied in neural modelling by Ricciardi et al. (1983). Giorno et al. (1989) introduced an algorithm which can be extended to any diffusion process whose transition density is known.

For the solution of the FPT problem when the transition density is not available there exists Siegert's equation for the Laplace transform $g_{S}\left(\lambda \mid x_{0}\right)$ of the FPT density,

$$
\frac{1}{2} \sigma^{2}\left(x_{0}\right) \frac{\partial^{2} g_{S}\left(\lambda \mid x_{0}\right)}{\partial x_{0}^{2}}+\mu\left(x_{0}\right) \frac{\partial g_{S}\left(\lambda \mid x_{0}\right)}{\partial x_{0}}-\lambda g_{S}\left(\lambda \mid x_{0}\right)=0
$$


with initial condition $g_{S}(\lambda \mid S)=1$ and $g_{S}\left(\lambda \mid x_{0}\right)<+\infty$ for each $x_{0}$. Then the standard relation

$$
M_{n}\left(S \mid x_{0}\right)=\left.(-1)^{n} \frac{\partial^{n} g_{S}\left(\lambda \mid x_{0}\right)}{\partial \lambda^{n}}\right|_{\lambda=0}
$$

can be used to obtain the moments $M_{n}\left(S \mid x_{0}\right)(n=1, \ldots)$ of the random variable $T_{S, x_{0}}$. Without a knowledge of the distribution (or its Laplace transform) for the FPT of the process $X$ through the threshold $S$, a recursion relation for the moments is available,

$$
\frac{1}{2} \sigma^{2}\left(x_{0}\right) \frac{\partial^{2} M_{n}\left(S \mid x_{0}\right)}{\partial x_{0}^{2}}+\mu\left(x_{0}\right) \frac{\partial M_{n}\left(S \mid x_{0}\right)}{\partial x_{0}}=-n M_{n-1}\left(S \mid x_{0}\right)
$$

and $M_{0}\left(S \mid x_{0}\right)=1, M_{n}(S \mid S)=0$, for $n=1, \ldots$

An alternative description of the diffusion process with coefficients $\mu(x)$ and $\sigma^{2}(x)$ is by the stochastic differential equation (Itô equation)

$$
\mathrm{d} X(t)=\mu(X(t)) \mathrm{d} t+\sigma(X(t)) \mathrm{d} W(t), X(0)=x_{0}
$$

where $W=\{W(t) ; t \geq 0\}$ is a standard Wiener process with zero mean, variance $t$ and $W(0)=0$. While the previous approach is oriented towards distributional properties of the model, this approach studies the sample paths and this type of description has simpler intuitive interpretation being an extension of deterministic model. Under some regularity conditions, the solution of (1.13) with given initial condition is unique and whenever there is a lack of the analytical solution, it can be simulated. The sophisticated methods for simulation of a diffusion process can be found in Rümelin (1982), Pardoux and Talay (1985), Kloeden and Platen (to appear) and the simulation in neuronal context is described by Musila and Lánský (in press). Before starting the simulation of the equation (1.13), one has to realize if it does not need to be transformed into the equation of Stratonovitch type. The equivalent of the Itô-type equation (1.13) is the Stratonovitch-type equation

$$
\mathrm{d} X(t)=\mu_{S}(X(t)) \mathrm{d} t+\sigma(X(t)) \mathrm{d} W(t) ; \quad X(0)=x_{0}
$$

where

$$
\mu_{S}=\mu-\frac{\sigma}{2} \frac{\mathrm{d} \sigma}{\mathrm{d} x}
$$

There is no difference between (1.13) and (1.14) for Ornstein-Uhlenbeck process as the infinitesimal 
variance (1.3) is constant for this model. For simplicity we mention only an equidistant time discretization with $0=\tau_{0}<\tau_{1}<\ldots<\tau_{n}$, with step size $h=\tau_{i+1}-\tau_{i}$. The simplest discrete time approximation is the stochastic analogue of the Euler scheme. For (1.13) it takes the form

$$
X_{i+1}=X_{i}+\mu\left(X_{i}\right) h+\sigma\left(X_{i}\right) \Delta W_{i} ; \quad X(0)=x_{0}
$$

where $\Delta W_{i}$ are independent and normally distributed random variables, $\Delta W_{i} \sim N(0, h)$ which can be generated by standard, e.g., Box-Mueller procedure. It transforms a pair $\left(U_{1}, U_{2}\right)$ of independent random variables uniformly distributed on $[0,1]$ into a pair $\left(N_{1}, N_{2}\right)$ of independent standard $N(0,1)$ - normally distributed random variables.

$$
N_{1}=\sqrt{-2 \log U_{1}} \cos \left(2 \pi U_{2}\right), N_{2}=\sqrt{-2 \log U_{1}} \sin \left(2 \pi U_{2}\right)
$$

The generators of $[0,1]$ uniformly dîstributed random variables belong to any standard software.

\section{Diffusion Approximation}

Stein's model with reversal potentiais can be given by the stochastic differential equation

$$
\mathrm{d} X=-\frac{1}{\tau} X \mathrm{~d} t+a\left(V_{E}-X\right) \mathrm{d} N^{+}(t)+i\left(X-V_{I}\right) \mathrm{d} N^{-}(t), \quad X(0)=x_{0}
$$

where $\tau>0,-1<i<0<a<1$ are constants; $N^{+}=\left\{N^{+}(t), t \geq 0\right\}, N^{-}=\left\{N^{-}(t), t \geq 0\right\}$ are two independent homogeneous Poisson processes with $N^{+}(0)=N^{-}(0)=0$ and intensities $\lambda$, $\omega$ resp.; $V_{I}$ $<x_{0}$ is a constant representing the inhibitory reversal potential and $V_{E}>x_{0}$ stands for another constant, the excitatory reversal potential. Due to the above mentioned transformation of the resting level to zero, we have $V_{I}<0<S<V_{E}$, where $S$ is the threshold depolarization. Let us also define Stein's model to permit us a comparison of both models:

$$
\mathrm{d} X=-\frac{1}{\tau} X \mathrm{~d} t+a_{E} \mathrm{~d} N^{+}(t)+i_{I} \mathrm{~d} N-(t), \quad X(0)=x_{0}
$$

where $i_{I}<0<a_{E}$ are constants; $\tau, x_{0}, S$ and the Poisson inputs are as before. In model (2.1) the jumps, which simulate postsynaptic potentials (PSPs), caused by the input are state-dependent in such a way that their magnitudes decrease linearly as $X$ approaches the boundaries $V_{I}, V_{E}$ resp. Hence the 
process cannot go below the value of the inhibitory reversal potential $V_{I}$ remaining confined within the boundaries $V_{I}$ and $V_{E}$. This is the main qualitative advantage of the model (2.1) over Stein's model (2.2). Tuckwell (1979) evaluated detailed comparison of these models.

As shown by Lánský and Lánská (1987) for the model (2.1) there is only deterministic limit for $a \rightarrow 0_{+}, i \rightarrow 0_{-}, \lambda \rightarrow+\infty$ and $\omega \rightarrow+\infty$. The simplest way how to overcome this disadvantage is to consider Stein's model with reversal potentials and random amplitudes of PSP

$$
\mathrm{d} X=-\frac{1}{\tau} X \mathrm{~d} t+(a+A)\left(V_{E}-X\right) \mathrm{d} N^{+}(t)+(i+I)\left(X-V_{I}\right) \mathrm{d} N^{-}(t), \quad X(0)=x_{0}
$$

where the interpretation of the parameters is the same as for (2.1) and additionally $A$ and $I$ are random variables, $\mathrm{E}(A)=\mathrm{E}(I)=0$, defined on the interval $(-a, 1-a)$, resp., $(-1-i,-i)$. One possibility is to consider Beta distribution transformed on the corresponding intervals for PSP random parts. From the biological point of view (2.3) is a more realistic model than (2.1) as there exists a great variability in the PSP contribution to the membrane potential due to the different positions of synaptic endings on the soma or dendrites of neuron. The attenuation of the PSP amplitude as it spreads from the input site to the trigger zone has been often studied and this phenomenon has been also several times included into the one-dimensional neuronal models (Stein, 1967; Vasudevan and Vittal, 1982; Musila and Lánský, 1992). Performing the diffusion approximation on the model (2.3) we get a diffusion process for which the reversal potentials are regular boundaries and to prevent the membrane potential from leaving the interval $\left(V_{I}, V_{E}\right)$ the additional boundary conditions have to be imposed (Hanson and Tuckwell, 1983; Kallianpur and Wolpert, 1987). In this way the main advantage of the model with reversal potentials over the classical models (Stein's, Ornstein-Uhlenbeck) is lost. To overcome this defect a slight modification of the model (2.3) has to be taken into consideration.

One way how to do it is to assume that the random part of PSP depends on both reversal potential simultaneously. Then the model leading to the diffusion approximation we are looking for can be written in the form

$$
\begin{gathered}
\mathrm{d} X=-\frac{1}{\tau} X \mathrm{~d} t+\left[a\left(V_{E}-X\right)+A\left(V_{E}-X\right)^{p}\left(X-V_{I}\right)^{q}\right] \mathrm{d} N^{+}(t)+ \\
\quad+\left[i\left(X-V_{I}\right)+I\left(V_{E}-X\right)^{q}\left(X-V_{I}\right)^{p}\right] \mathrm{d} N^{-}(t), \quad X(0)=x_{0}
\end{gathered}
$$


where $p>0, q \geq 0$ are constants. Note that for $p=1$ and $q=0$ the models (2.4) and (2.3) coincide. Denoting $\Delta X(t)=X(t+\Delta t)-X(t)$, we can compute the infinitesimal moments defined by (1.2) and (1.3),

$$
M_{1}(x)=\lim _{\Delta t \rightarrow 0_{+}} \frac{\mathrm{E}(\Delta X(t) \mid X(t)=x)}{\Delta t}=-x / \tau+\lambda a\left(V_{E}-x\right)+\omega i\left(x-V_{I}\right)
$$

and

$$
\begin{gathered}
M_{2}(x)=\lim _{\Delta t \rightarrow 0_{+}} \frac{\mathrm{E}\left(\Delta X(t)^{2} \mid X(t)=x\right)}{\Delta t}=\lambda a^{2}\left(V_{E}-x\right)^{2}+\omega i^{2}\left(x-V_{I}\right)^{2}+ \\
+\lambda \mathrm{E}\left(A^{2}\right)\left(V_{E}-x\right)^{2 p}\left(x-V_{I}\right)^{2 q}+\omega \mathrm{E}\left(I^{2}\right)\left(V_{E}-x\right)^{2 q}\left(x-V_{I}\right)^{2 p}
\end{gathered}
$$

Now, for a sequence of models (2.4) indexed by $n$ we assume $a_{n} \rightarrow 0_{+}, i_{n} \rightarrow 0_{-}, \lambda_{n} \rightarrow+\infty, \omega_{n} \rightarrow$ $+\infty$ in such a way that $a_{n} \lambda_{n} \rightarrow \mu \geq 0, i_{n} \omega_{n} \rightarrow \nu \leq 0$ and simultaneously $\mathrm{E}\left(A_{n}^{2}\right) \rightarrow 0_{+}, \mathrm{E}\left(I_{n}^{2}\right) \rightarrow$ $0_{+}$, ensuring that $\lambda_{n} \mathrm{E}\left(A_{n}^{2}\right) \rightarrow \sigma_{A}^{2}>0, \omega_{n} \mathrm{E}\left(I_{n}^{2}\right) \rightarrow \sigma_{I}^{2}>0$. In this way we may consider, as $M_{j}(x) \rightarrow$ 0 for $j>2$, a diffusion process specified by infinitesimal mean following from (2.5),

$$
\mu(x)=-x / \tau+\mu\left(V_{E}-x\right)+\nu\left(x-V_{I}\right)
$$

and by infinitesimal variance derived from (2.6)

$$
\sigma^{2}(x)=\sigma_{A}^{2}\left(V_{E}-x\right)^{2 p}\left(x-V_{I}\right)^{2 q}+\sigma_{I}^{2}\left(V_{E}-x\right)^{2 q}\left(x-V_{I}\right)^{2 p}
$$

The formal diffusion approximation of $(2.4)$ by (2.7) and (2.8) was presented by Lánská (1988). The model (2.4) can be simplified in such a way that the fluctuation of either excitatory PSP or inhibitory PSP is neglected. Which one of PSP would be more suitable to be considered as non-random is questionable. However, due to the experimental values of $V_{I}$ and $V_{E}$, ( the effect of excitatory reversal potential is smaller when compared with the effect of the inhibitory one) it seems to be more appropriate to assume

$$
\mathrm{d} X=-\frac{1}{\tau} X \mathrm{~d} t+a\left(V_{E}-X\right) \mathrm{d} N^{+}(t)+\left[i\left(X-V_{I}\right)+I\left(V_{E}-X\right)^{q}\left(X-V_{I}\right)^{p}\right] \mathrm{d} N^{-}(t)
$$

and it gives qualitatively the same limiting diffusion model as (2.4). The open problem which remains is to chose the values of parameters $p$ and $q$. From the point of view of the model tractability it seems to 
be suitable to assign $p=q=1 / 2$. Thus we consider the diffusion model

$$
\mathrm{d} X(t)=\left(-X / \tau+\mu\left(V_{E}-X\right)+\nu\left(X-V_{I}\right)\right) \mathrm{d} t+\sigma \sqrt{\left(V_{E}-X\right)\left(X-V_{I}\right)} \mathrm{d} W(t)
$$

where $\sigma_{\mathrm{A}}^{2}+\sigma_{\mathrm{I}}^{2}=\sigma^{2}>0$. One may object replacing the model (2.3) by (2.4), resp., by (2.9). Up to our knowledge there is neither experimental support nor an evidence against it. However, the modification is rather formal as for the diffusion approximation the variances of $A$ and $I$ tend to zero and thus these terms can be made negligibly small.

One of the alternatives to the model $(2.10)$ is that in which the inhibitory reversal potential plays a specific role,

$$
\mathrm{d} X(t)=\left(-X / \tau+\mu\left(V_{E}-X\right)+\nu\left(X-V_{I}\right)\right) \mathrm{d} t+\sigma \sqrt{\left(X-V_{I}\right)} \mathrm{d} W(t)
$$

The process (2.11), called the Feller process, can be derived either from (2.9) assuming that the random part of IPSP is independent of excitatory reversal potential, i.e., $q=0, p=1 / 2$, or using a different scenario as done by Giorno et al. (1988). In their paper detailed description of the model (2.11) is presented. Balossino et al. (1992) compared the Ornstein-Uhlenbeck and the Feller models discussing how to determine the values of the parameters for such purpose.

Finally, let us also remind Ornstein-Uhlenbeck process as the limiting diffusion of the Stein's model (2.1), (for formal derivation see Kallianpur (1983), Lánský (1984)):

$$
\mathrm{d} X(t)=(-X / \tau+\mu+\nu) \mathrm{d} t+\sigma \mathrm{d} W(t)
$$

There exists an enormous number of papers devoted to the the model (2.12). In neuronal context, it was analysed mainly by Capocelli and Ricciardi (1971), Sato (1978), Ricciardi and Sacerdote (1979), Wan and Tuckwell (1982).

The effect of the inclusion of reversal potentials into the diffusion models is apparent when comparing (2.12) with (2.10) or (2.11). From qualitative point of view it means that the infinitesimal variance becomes non-constant while the drift preserves its linearity. However, the parameters in the drift term are qualitatively entirely different. There is constant "leakage term" $-\tau^{-1}$ in $(2.12)$ while for 
the models with reversal potentials the leakage is input dependent $\left(-\tau^{-1}+\mu+\nu\right)$. Also the absolute term of the drift is multiplied by the reversal potentials in the models where these are considered.

\section{The Diffusion Model}

Before studying the FPT problem for the derived model (2.10) let us present some of its properties. In this part we do not take into account that crossing $S<V_{E}$ terminates the process. Nevertheless, we restrict ourselves mainly on the question what is the behavior of the derived diffusion neuronal model at $V_{I}$ as the boundary $V_{E}>S$, and the threshold $S$ is an absorbing barrier for which we aim to solve FPT problem. It is convenient to transform the process $X(t)$ into a process $Y(t)$ defined on the interval $(0,1)$ using the transformation $y=k(x)=\left(x-V_{I}\right) /\left(V_{E}-V_{I}\right)$. We get from (1.7) and (1.8) that

$$
\mathrm{d} Y(t)=(-\alpha Y+\beta) \mathrm{d} t+\sigma \sqrt{(1-Y) Y} \mathrm{~d} W(t), \quad Y(0)=y_{0}=\frac{x_{0}-V_{I}}{V_{E}-V_{I}},
$$

where $\alpha=1 / \tau+\mu-\nu>\beta=\mu-V_{I} /\left(\tau\left(V_{E}-V_{I}\right)\right)>0$. Comparing (2.7) and (3.1) we can see that the infinitesimal mean of the process stays linear under the transformation. The model specified by (3.1) resembles the model of genetical changes in the presence of mutation pressure (Goel and Richter-Dyn, 1974). In the same way as for the genetical model we may deduce the boundary behavior for our model. For $\beta<\sigma^{2} / 2$ the boundary $V_{I}$ is regular, otherwise it is an entrance boundary. The regularity of the boundary is the feature of the model we aim to avoid for the reasons presented above. As $\mu$ is positive, the sufficient condition for $V_{I}$ to be the entrance boundary is

$$
\sigma^{2}<\frac{-2 V_{I}}{\tau\left(V_{E}-V_{I}\right)}
$$

The boundary $V_{E}$ is regular for $\alpha-\beta<\sigma^{2} / 2$. Analogously to the previous case, we deduce that the sufficient condition for the excitatory reversal potential to be the entrance boundary is

$$
\sigma^{2}<\frac{2 V_{E}}{\tau\left(V_{E}-V_{I}\right)}
$$

In general, unattainability of the boundaries depends on $\sigma^{2}$ in that way that for higher values of $\mu$, respectively $\nu$ in the case of boundary $V_{E}, \sigma^{2}$ can be bigger. This result has a simple intuitive 
interpretation. The considered transformation set the resting level to $-V_{I} /\left(V_{E}-V_{I}\right)$ which can be the standard reset value. On the other hand if the reset $y_{0}=0$ is taken into account, it means for $X$ that $X(0)=V_{I}$ and it corresponds to the reset below the resting level, for example due to the afterhyperpolarization.

The transition probability density function $f_{y_{0}}(y, t)$ which fulfills (1.4) and (1.5), for the process $Y$, can be written as an infinite series of hypergeometric functions (Goel and Richter-Dyn, 1974). We will use the following notation: $\phi=2 \alpha / \sigma^{2}, \xi=2 \beta / \sigma^{2}, \lambda_{i}=i\left(2 \alpha+(i-1) \sigma^{2}\right) / 2, \epsilon=\phi-\xi-1, b=\xi$ $-1, \tilde{F}_{i}(y)=F(\phi+i-1,-i, \phi-\xi ; y), F$ is a hypergeometric function and $P_{i}^{\epsilon, b}(y)$ is a Jacobi polynomial, see Appendix A. Then

$$
f_{y_{0}}(y, t)=y^{\xi-1}(1-y)^{\phi-\xi-1} \sum_{i=0}^{\infty} \frac{(\phi+2 i-1) \Gamma(\phi+i-1) \Gamma(\phi-\xi+i)}{i ! \Gamma^{2}(\phi-\xi) \Gamma(\xi+i)} \tilde{F}_{i}(1-y) \tilde{F}_{i}\left(1-y_{0}\right) \mathrm{e}^{-\lambda_{i} t}=
$$

$$
=y^{\xi-1}(1-y)^{\phi-\xi-1} \sum_{i=0}^{\infty} \frac{(\phi+2 i-1) \Gamma(\phi+i-1)}{\Gamma(\phi-\xi) \Gamma(\xi+i)} P_{i}^{\epsilon, b}(2 y-1) \tilde{F}_{i}\left(1-y_{0}\right) \mathrm{e}^{-\lambda_{i} t}
$$

To compute the moments for the studied process, it is useful to have the relation for the generating function. Let

$$
H_{y_{0}}(s, t)=\int_{0}^{1} \mathrm{e}^{s y_{y_{0}}(y, t) \mathrm{d} y,}
$$

then using (3.4) we obtain

$$
\begin{gathered}
H_{y_{0}}(s, t)=\sum_{i=0}^{\infty} \frac{(\phi+2 i-1)}{\Gamma(\phi-\xi) \Gamma(\xi+i)} \tilde{F}_{i}\left(1-y_{0}\right) \mathrm{e}^{-\lambda_{i} t} \frac{\Gamma(\phi-\xi+i)}{i !} \times \\
\sum_{m=0}^{i} \frac{(-1)^{m_{i} !}}{m !(i-m) !} \frac{\Gamma(\phi-1+i-m)}{\Gamma(\phi-\xi+m)} B(\xi, m+\phi-\xi) M(\xi, m+\phi, s),
\end{gathered}
$$

where $\Gamma(a)$ is a Gamma function, $B(a, b)$ is Beta function and $M(a, b, s)$ is a Kummer's function, see Appendix A. From (3.5) we get the mean of the process $Y$ in the form

$$
\mathrm{E}\left(Y(t) \mid Y(0)=y_{0}\right)=\left.\frac{\partial H_{y_{0}}(s, t)}{\partial s}\right|_{s=0}=\frac{x_{0}-V_{I}}{V_{E}-V_{I}} \mathrm{e}^{-\alpha t}+\frac{\beta}{\alpha}\left(1-\mathrm{e}^{-\alpha t}\right)
$$


and using the inverse transformation $k^{-1}$ the mean of the process $X$ is

$$
\mathrm{E}\left(X(t) \mid X(0)=x_{0}\right)=x_{0} \mathrm{e}^{-\alpha t}+\frac{\mu V_{E}-\nu V_{I}}{\alpha}\left(1-\mathrm{e}^{-\alpha t}\right)
$$

with the asymptotic level as $t \rightarrow+\infty$,

$$
\mathrm{E}(X(+\infty))=\frac{\mu V_{E}-\nu V_{I}}{\alpha}
$$

How fast the process get to the asymptotic level (3.8) is controlled by $\alpha$ and in general it is faster than for Ornstein-Uhlenbeck process, respectively the original Stein's model, as there it depends only on $1 / \tau$. The input dependent "leakage rate" is one of the consequences of the inclusion of reversal potentials. Of course, all the models $(2.1),(2.3),(2.4),(2.10)$ and that specified by (2.7) and (2.8) are characterized by the same functional form for the mean trajectory (3.7). The second moment can be again copmuted from $(3.5)$ :

$$
\begin{gathered}
\mathrm{E}\left(Y^{2}(t) \mid Y(0)=y_{0}\right)=\left.\frac{\partial^{2} H_{y_{0}}(s, t)}{\partial s^{2}}\right|_{s=0}= \\
=\frac{\xi(\xi+1)}{\phi(\phi+1)}+\mathrm{e}^{-\alpha t} \frac{2(\phi-\xi)(\xi+1)}{\phi(\phi+2)} \tilde{F}_{1}\left(1-y_{0}\right)+\mathrm{e}^{-\left(2 \alpha+\sigma^{2}\right) t} \frac{(\phi-\xi)(v-\xi-1)}{(\phi+1)(\phi+2)} \tilde{F}_{2}\left(1-y_{0}\right) .
\end{gathered}
$$

and thus the stationary second moment is

$$
\mathrm{E}\left(Y^{2}(+\infty)\right)=\frac{\beta\left(2 \beta+\sigma^{2}\right)}{\alpha\left(2 \alpha+\sigma^{2}\right)}
$$

from which we can easily compute the variance of the limiting membrane potential

$$
\operatorname{Var}(X(+\infty))=\operatorname{Var}(Y(+\infty))\left(V_{E}-V_{I}\right)^{2}=\frac{\beta(\alpha-\beta) \sigma^{2}}{\alpha^{2}\left(2 \alpha+\sigma^{2}\right)}\left(V_{E}-V_{I}\right)^{2}
$$

Some information about the character of the membrane potential can be deduced from its stationary distribution (Hanson and Tuckwell, 1983). Solving the corresponding differential equation or substituting directly into (1.6) we derive

$$
f(x)=\frac{\Gamma(B+A)}{\Gamma(B) \Gamma(A)}\left(V_{E}-V_{I}\right)^{1-A \cdot B}\left(V_{E}-x\right)^{A-1}\left(x-V_{I}\right)^{B-1}
$$


where $A=\frac{2\left(V_{E} / \tau-\nu\left(V_{E}-V_{I}\right)\right)}{\sigma^{2}\left(V_{E}-V_{I}\right)}>0$ and $B=\frac{2\left(-V_{I} / \tau+\mu\left(V_{E}-V_{I}\right)\right)}{\sigma^{2}\left(V_{E}-V_{I}\right)}>0$. The shape of the distribution (3.12) is that of a Beta distribution. Under the condition (3.2) it holds that $B>1$ and (3.3) ensures $A>1$. These ranges of $A$ and $B$ induces that (3.12) is single peaked with peak at

$$
x_{m}=\frac{V_{E}(B-1)-V_{I}(1-A)}{B+A-2}
$$

and it means that the mode of the membrane potential is inside the interval formed by the reversal potentials. The distribution (3.12) is symmetrical with mode at $x_{m}=\left(V_{E}-V_{I}\right) / 2$ under the codition $A$ $=B$. The mean and variance of the distribution (3.12) are given by (3.8) and (3.11). Obviously, for $\sigma^{2}$ $\rightarrow 0$ the distribution (3.12) becomes degenerated at $E(X(+\infty))$.

If we linearly transform the process $X$ to $(-1,1)$, (using the transformation $y=k(x)=-1+$ $\left.2 x /\left(V_{E}-V_{I}\right)\right)$ the spectral expansion of the transition density is available in terms of Jacobi polynomials (Karlin and Taylor, $1 \overline{98} 1 ;$ p.335).

\section{Properties of the Firing Times}

While for the properties of the trajectories it was necessary to transform process $Y$ back to $X$, the properties of FPT are not changed by the state-space transformation. Having at disposal the transition density (3.4), we could use equation (1.9) for numerical evaluation of FPT density as we are not able to find its analytical solution. On the other hand, the moments of the FPT can be computed. Using the form of infinitesimal moments employed in (3.1), the equation (1.10) can be identified with the Gaussian equation (Abramowitz and Stegun, 1965)

$$
\left(y_{0}-1\right) y_{0} \frac{\partial^{2} g_{S}\left(\lambda \mid y_{0}\right)}{\partial y_{0}^{2}}+\left[\gamma-(\kappa+\theta+1) y_{0}\right] \frac{\partial g_{S}\left(\lambda \mid y_{0}\right)}{\partial y_{0}}-\kappa \theta g_{S}\left(\lambda \mid y_{0}\right)=0
$$

which has a general solution

$$
g_{S}\left(\lambda \mid y_{0}\right)=C_{1} F\left(\kappa, \theta, \gamma ; y_{0}\right)+C_{2} y_{0}{ }^{1-\gamma} F\left(\kappa+1-\gamma, \theta+1-\gamma, 2-\gamma ; y_{0}\right)
$$

where $F\left(\kappa, \theta, \gamma ; y_{0}\right)$ is a hypergeometric function (see Appendix A). For a specific solution we have to identify values for the parameters appearing in (4.1); in our case $\gamma=2 \beta / \sigma^{2}, \kappa+\theta+1=2 \alpha / \sigma^{2}$ and $\kappa \theta=$ $2 \lambda / \sigma^{2}$. The constant $C_{2}$ equals to 0 using arguments given in Appendix B and the constant $C_{1}$ follows 
from the initial condition $g_{S}(\lambda \mid S)=1$. Thus

$$
g_{S}\left(\lambda \mid y_{0}\right)=\frac{F\left(\kappa, \theta, \gamma ; y_{0}\right)}{F(\kappa, \theta, \gamma ; S)}
$$

and due the the form of hypergeometric function we can prove (see Ricciardi and Sato, 1990, p. 280) that the FPT density $g_{S}\left(t \mid y_{0}\right)$ can be written as a sum of exponential functions. For the threshold $S$ close to one (the original threshold close to excitatory reversal potential), the result of Nobile et al. (1985) can be used. It holds

$$
g_{S}\left(t \mid y_{0}\right) \approx \frac{1}{M_{1}\left(S \mid y_{0}\right)} \exp \left(-\frac{t}{M_{1}\left(S \mid y_{0}\right)}\right)
$$

and it follows from it that the ISIs form Poisson process.

Substituing (4.3) into (1.11) the first moment, $\mathrm{E}\left(T_{S, y_{0}}\right)=M_{1}\left(S \mid y_{0}\right)$, equals

$$
M_{1}\left(S \mid y_{0}\right)=\frac{\partial}{\partial \lambda}\left(\left.F\left(\kappa, \theta, \overline{\gamma ; S)}-F\left(\kappa, \theta, \gamma ; y_{0}\right)\right)\right|_{\lambda=0}=\frac{1}{\beta} \sum_{n=0}^{\infty} \frac{\left(2 \alpha / \sigma^{2}\right)_{n}}{\left(2 \beta / \sigma^{2}\right)_{n}} \frac{S^{n+1}-y_{0}^{n+1}}{\beta(n+1)}\right.
$$

where $(a)_{n}=a(a+1) \ldots(a+n-1),(a)_{0}=1$. How to derive $M_{1}\left(S \mid y_{0}\right)$ using (1.12) is shown in Appendix B. For $\sigma^{2} \rightarrow 0$ the first moment $M_{1}\left(S \mid y_{0}\right)$ converges to the solution of equation $\mathrm{E}(Y(t) \mid Y(0)=$ $\left.y_{0}\right)=S$. Using again (4.3) in (1.11), the second moment is

$$
\begin{array}{r}
M_{2}\left(S \mid y_{0}\right)=\left.\frac{\partial^{2}}{\partial \lambda^{2}}\left(F\left(\kappa, \theta, \gamma ; y_{0}\right)-F(\kappa, \theta, \gamma ; S)\right)\right|_{\lambda=0}- \\
-\left.2 \frac{\partial}{\partial \lambda}\left(F\left(\kappa, \theta, \gamma ; y_{0}\right)-F(\kappa, \theta, \gamma ; S)\right)\right|_{\lambda=0} \times\left.\frac{\partial}{\partial \lambda}(F(\kappa, \theta, \gamma ; S))\right|_{\lambda=0},
\end{array}
$$

where

$$
\left.\frac{\partial}{\partial \lambda}(F(\kappa, \theta, \gamma ; y))\right|_{\lambda=0}=\frac{1}{\beta} \sum_{n=0}^{\infty} \frac{\left(2 \alpha / \sigma^{2}\right)_{n}}{\left(2 \beta / \sigma^{2}+1\right)_{n}} \frac{y^{n+1}}{(n+1)}
$$

and

$$
\left.\frac{\partial^{2}}{\partial \lambda^{2}}(F(\kappa, \theta, \gamma ; y))\right|_{\lambda=0}=\frac{8 \alpha}{\beta\left(2 \beta+\sigma^{2}\right)} \sum_{n=0}^{\infty} \frac{\left(2 \alpha / \sigma^{2}+1\right)_{n} y^{n+2}}{\left(2 \beta / \sigma^{2}+2\right)_{n}(n+2)}\left(\sum_{j=1}^{n+1} \frac{1}{j\left(2 \alpha+\sigma^{2}(j-1)\right)}\right)
$$

Thus substituing into (4.6) we obtain 


$$
\begin{gathered}
M_{2}\left(S \mid y_{0}\right)=2 M_{1}\left(S \mid y_{0}\right) \frac{1}{\beta} \sum_{n=0}^{\infty} \frac{\left(2 \alpha / \sigma^{2}\right)_{n}}{\left(2 \beta / \sigma^{2}+1\right)_{n}} \frac{S^{n+1}}{(n+1)}- \\
-\frac{8 \alpha}{\beta\left(2 \beta+\sigma^{2}\right)} \sum_{n=0}^{\infty} \frac{\left(2 \alpha / \sigma^{2}+1\right)_{n} S^{n+2}-y_{0}^{n+2}}{\left(2 \beta / \sigma^{2}+2\right)_{n}(n+2)}\left(\sum_{j=1}^{n+1} \frac{1}{j\left(2 \alpha+\sigma^{2}(j-1)\right)}\right)
\end{gathered}
$$

and

$$
\begin{gathered}
\operatorname{Var}\left(T_{S, y_{0}}\right)=M_{1}\left(S \mid y_{0}\right) \frac{1}{\beta} \sum_{n=0}^{\infty} \frac{\left(2 \alpha / \sigma^{2}\right)_{n}}{\left(2 \beta / \sigma^{2}+1\right)_{n}} \frac{S^{n+1}+y_{0}^{n+1}}{(n+1)}- \\
-\frac{8 \alpha}{\beta\left(2 \beta+\sigma^{2}\right)} \sum_{n=0}^{\infty} \frac{\left(2 \alpha / \sigma^{2}+1\right)_{n} S^{n+2}-y_{0}^{n+2}}{\left(2 \beta / \sigma^{2}+2\right)_{n}(n+2)}\left(\sum_{j=1}^{n+1} \frac{1}{j\left(2 \alpha+\sigma^{2}(j-1)\right)}\right)
\end{gathered}
$$

which enables us to compute the coefficient of variation CV often used in the theoretical as well as experimental studies on neuronal firing and defined as $\mathrm{CV}=\sqrt{\operatorname{Var}\left(T_{S, y_{0}}\right)} / \mathrm{E}\left(T_{S, y_{0}}\right)$. Of course, the moments (4.5) and (4.7) have to fulfill the equation (1.12). One can hardly expect that the inverse Laplace transform of the function $g_{S}\left(\lambda \mid y_{0}\right)$ given by (4.3) is available and thus only the approximation methods may solve this problem. On the other hand, the moments $M_{j}\left(S \mid y_{0}\right)$ for $j>2$ can be evaluated.

\section{Methods for Approximations}

Now, for the sake of simplicity we will approximate the function $F(\kappa, \theta, \gamma ; y)$ only by two first members, i.e.

$$
g_{S}\left(\lambda \mid y_{0}\right)=\frac{1+\frac{\lambda}{\beta} y_{0}\left(1+\frac{\lambda+\alpha}{2 \beta+\sigma^{2}} y_{0}\right)}{1+\frac{\lambda}{\beta} S\left(1+\frac{\lambda+\alpha}{2 \beta+\sigma^{2}} S\right)}=\frac{P(\lambda)}{Q(\lambda)}
$$

Under the condition $\lambda S+\sigma^{2} / 2>\beta$ (it is always true for $S$ sufficiently high), the polynom $Q(\lambda)$ has two different negative roots $\lambda_{1}$ and $\lambda_{2}$. Then $g_{S}\left(\lambda \mid y_{0}\right)$ can be inverted and $g_{S}\left(t \mid y_{0}\right)$ is a sum of two exponential functions.

Then mean and variance can be approximated by

$$
\mathrm{E}\left(T_{S, y_{0}}\right)=\frac{S-y_{0}}{\beta}\left(1+\frac{\alpha\left(S+y_{0}\right)}{2 \beta+\sigma^{2}}\right)
$$

and 


$$
\operatorname{Var}\left(T_{S, y_{0}}\right)=\frac{S-y_{0}}{\beta^{2}\left(2 \beta+\sigma^{2}\right)}\left\{\left(S+y_{0}\right) \sigma^{2}+2\left(S^{2}+S y_{0}+y_{0}^{2}\right) \alpha+\frac{\left(S^{3}+S^{2} y_{0}+S y_{0}^{2}+y_{0}^{3}\right) \alpha^{2}}{2 \beta+\sigma^{2}}\right\}
$$

Then the approximation for coefficient of variation equals

$$
\mathrm{CV}=\frac{\left(2 \beta+\sigma^{2}\right)\left(\left(S+y_{0}\right) \sigma^{2}+2\left(S^{2}+S y_{0}+y_{0}^{2}\right) \alpha+\left(S^{3}+S^{2} y_{0}+S y_{0}^{2}+y_{0}^{3}\right) \alpha^{2}\right)}{\left(S-y_{0}\right)\left(2 \beta+\sigma^{2}+\alpha\left(S+y_{0}\right)\right)^{2}}
$$

Some information about the FPT can be obtained from the moments of $X$, namely the mean membrane potential trajectory. One way of approximating the mean interspike interval $\mathrm{E}\left(T_{S, x_{0}}\right)$ is to use the time $t^{*}$, taken for the mean voltage given by (3.7) to cross the threshold. The approximation $t^{*}$ will be finite if the asymptotic mean membrane potential (3.8) is higher than the threshold,

$$
\left(\mu V_{E}-\nu V_{I}\right) \alpha^{-1}>S
$$

Under this condition, setting $\mathrm{E}(X(t))=S$ and then solving it for $t$, we obtain

$$
t^{*}=-\alpha^{-1} \ln \left(\frac{S \alpha-\left(\mu V_{E}-\nu V_{I}\right)}{x_{0} \alpha-\left(\mu V_{E}-\nu V_{I}\right)}\right)
$$

The reliability of the approximation (5.6) depends on the variance of the process $X$ which can computed from (3.9). The model neuron fires more and more regularly at intervals (5.6) if the asymptotic mean membrane potential (3.8) increases relatively to the asymptotic variance (3.11).

The condition (5.5) for application of (5.6) is modified if the spontaneous decay of the membrane potential disappears, $\tau=+\infty$. Then the ratio between the input intensities and the ratio between the relative distances of the threshold from the reversal potentials has to fulfil the condition $\mu / \nu$ $<\left(S-V_{I}\right) /\left(V_{E}-S\right)$. The approximation for the mean firing time gives an interesting comparison with Stein's original model (2.2). If we set $b_{E}=a V_{E}$ and $b_{I}=-i V_{I}$, which means that the jump sizes in the model (2.2) are the same as the jump sizes in the model (2.1) at the resting level, then the approximation for the mean firing time, $\tilde{t}$, is given by

$$
\tilde{t}=-\tau \ln \left(\frac{S-\tau\left(\mu V_{E}-\nu V_{I}\right)}{x_{0}-\tau\left(\mu V_{E}-\nu V_{I}\right)}\right)
$$

Paralleling the arguments in Smith and Smith (1984), $\tilde{t}$ may be greater or smaller than the corresponding estimate, $t^{*}$, from the above reversal potential models. For a given $\tau, \mu, \nu, V_{E}, V_{I}$, if $\tilde{t}$ 
$>t_{c}$, then $\tilde{t}<t^{*}$ and if $\tilde{t}<t_{c}$, then $\tilde{t}>t^{*}$ where $t_{c}$ is the time at which the mean voltage trajectories for the two models intersect. Of course, we have assumed that the asymptotic level of the mean voltage in the reversal potential model is greater than $S$ so that the approximation method is applicable.

\section{Numerical Results and Discussion}

The parameters of the model are of two types; input dependent and input independent. Those not depending on the neuronal input, despite their variability with the type of neuron as well as with internal conditions, are relatively more stable. For tentative identification of their values we may refer to the neurophysiological handbooks. For example, there is no doubt that $\left|V_{I}\right|<\left|V_{E}\right|$ and thus just the condition (3.2) need to be considered. On the other hand, for the input parameters, namely $\mu, \nu$, and $\sigma$, the estimation from experimental data is the only way how to find their values. Therefore, in the following examples, the parameters of input are considered as variable.

The values used in the previous studies performed by Tuckwell (1979), Wilbur and Rinzel (1983) and also by Lánský and Musila (1991) have been applied here to achieve some comparability of the results. Assuming the resting potential to be transformed to zero and the resetting potential being also zero, i.e., $x_{0}=0 \mathrm{mV}$, the reversal potentials were taken $V_{I}=-10 \mathrm{mV}, V_{E}=100 \mathrm{mV}$ and the threshold $S=10 \mathrm{mV}$. The parameter of spontaneous decay has been chosen $\tau=5.8 \mathrm{~ms}$,

\section{Example 1.}

The parameters controlling the PSP sizes and the intensities of the input processes in the model (2.1) were taken in the above cited papers: $i=-0.2, a=0.02, \lambda=8 / \tau \approx 1.379 \mathrm{~ms}^{-1}$ and $\omega=4 / \tau \approx$ $0.690 \mathrm{~ms}^{-1}$. Let us assume the values of the input parameters in the way the diffusion approximation has been constructed, $\mu=\lambda a=0.0276 \mathrm{~ms}^{-1}, \nu=\omega i=-0.138 \mathrm{~ms}^{-1}$. Therefore the time constant of the mean membrane potential equals $\alpha=0.338 \mathrm{~ms}^{-1}$ and we can see that $\mu$ contributes to it negligibly. The other constants take values $\beta=0.043 \mathrm{~ms}^{-1}$ and $\mu V_{E}-\nu V_{I}=1.38 \mathrm{mVms}^{-1}$. The asymptotic level of the membrane potential given by $(3.8)$ is $\mathrm{E}(X(+\infty))=4.083 \mathrm{mV}$. Its variance is a function of $\sigma^{2}$ as it follows from (3.12) and the derivation of (2.8) does not offer any approximation for $\sigma^{2}$ as those for $\mu$ and $\nu$. The only hint is to keep $V_{I}$ to be the entrance boundary which induces $\sigma^{2}<0.087$. More 
restrictive condition which does not consider the actual values of $\mu$ and $\nu$ can be found using (3.2) and it gives the range $\sigma^{2}<0.031$. Taking for example $\sigma^{2}=0.03$, then $\operatorname{Var}(X(+\infty))=57.40 \mathrm{mV}^{2}$ and the parameters of the distribution (3.12) can be computed, $A=19.65$ and $B=2.88$ which induces that $x_{m}$ $=0.097 \mathrm{mV}$. The probability density (3.12) specified by these parameters is illustrated on Fig. 1 . together with the simulated histograms of the membrane potential for different $t$; one thousand trajectories was simulated with the simulation step $h=0.02$ using the schema defined by (1.15) and (1.16). For the considered value of $\sigma^{2}$, the membrane potential fluctuates in a rather broad range, despite that this parameter seems to be small when compared with those values used in numerical studies on the Ornstein-Uhlenbeck model. It is only an illusion as here the infinitesimal variance depends on the state of the process and the coefficient at $d W$ in the equation (2.10) takes its maximal value in the mid distance between the reversal potentials, $\sqrt{0.03 \times 55^{2}}=1.65$.

The moments of the FPT for Stein's model with reversal potentials were computed by Wilbur and Rinzel (1983) and also by Lánský and Musila (1991), mean $=19.5 \mathrm{~ms}, \mathrm{CV}=0.88$. Using (5.2) with neglected second term in the parenthesis we get the mean approximated by $2.10 \mathrm{~ms}$. It is independent of $\sigma^{2}$ and thus reliable only for high values of this parameter. For the above specified $\sigma^{2}, \sigma^{2}=0.03,(5.2)$ gives the approximation $3.76 \mathrm{~ms}$. As the asymptotic membrane potential is below the threshold, the approximation (5.6) cannot be used and also (5.7) does not work for this set of parameters. From (4.5) we obtain $E\left(T_{10,0}\right)=6.34 \mathrm{~ms}$ and using (4.8) we compute $\mathrm{CV}=1.00$. We can see that for this choice of $\sigma^{2}$ the mean ISI for the diffusion model is shorter than for its discontinuous counterpart. Decreasing $\sigma^{2}$ the mean ISI becomes longer “(Fig. 2a.) which holds also for the Ornstein-Uhlenbeck model (compare with fig 3. of Ricciardi and Sacerdote, 1979). For the parameters employed in this example, the mean goes to infinity with $\sigma \rightarrow 0$. The means of FPT of Stein's model with reversal potential and the model (2.10) practically coincides for $\sigma^{2}=0.0063$. For this parameter in the studied diffusion model we have $\mathrm{E}\left(T_{10,0}\right)=19.34 \mathrm{~ms}$ and from (4.8) we obtain $\mathrm{CV}=0.87$. How CV depends on $\sigma$ is illustrated on Fig. 2 b.

The simulation of the FPT in the case of a process with continuous trajectories overestimates the actual values due to the discretization, (Musila and Lánský, in press). It is clear that between any two simulated values below the threshold, the trajectory could elevate above the threshold. With 
decreasing the simulation step this overestimation becomes smaller. We are not aware of any theoretical results connecting the overestimation and the step. Three simulation runs, each of them containing one thousand realisations, were performed for $h=0.02 ; 0.01$ and 0.005 with the means $6.85 \mathrm{~ms}, 6.80 \mathrm{~ms}$, $6.45 \mathrm{~ms}$ and CVs $0.97,1.01,1.01$, respectively. Both moments exhibit a trend to the correct values. The achieved histogram is illustrated on Fig. 3a. There is a striking similarity with exponential distribution also suggested by the value of $\mathrm{CV}$. The simulated data were tested against the hypotheses of exponential distribution with the theoretical mean (illustrated together with the histogram) and hypothesis about the fit cannot be rejected. We also simulated $(h=0.02)$ the ISIs using the parameter $\sigma^{2}=0.0063$ and we obtained mean $20.51 \mathrm{~ms}$ and CV 0.88 ; for the histogram see Fig. 3b. There is an apparent change of the shape caused by $\sigma$.

The example illustrated in Fig. 3a. shows that there exist sets of input parameters for which the output activity of the model neurōn is Poissonian. This just confirms the theoretical results and fits the experimental findings in many types of neurons. On the opposite side is the regular firing achieved when $\sigma$ tends to zero as shown in Fig. 2a. Note that this is not that type of regularity achieved by limiting the ISI with the absolute refractory period. Also this type of experimental findings is common. Between these two extremal types of activity (Poissonian and regular) is a broad spectrum of unimodal and positively skewed ISIs histograms such as illustrated in Fig. 3b. Both these two features ensure that the tails of the histograms posese an exponential-like form permiting the same approximationas made by Pacut and Dabrowski (1988) for the Ornstein-Uhlenbeck model.

\section{Example 2.}

Tuckwell (1979) compared the model (2.1) specified by parameters in Example 1. with the same model considering neglected inhibition, $\omega=0$. For the diffusion analog $(2.10)$ this means $\nu=0$. Now the time constant of the mean membrane potential equals $\alpha=0.20 \mathrm{~ms}^{-1}$ and we can see that it differs only slightly from $\tau^{-1}=0.173 \mathrm{~ms}^{-1}$ which is the time constant of Stein's model (2.2) where no reversal potentials are considered. Of course $\beta$ remains the same as in Example 1. and thus the condition on $\sigma^{2}$ to ensure $V_{I}$ to be the entrance boundary is identical as in the previous case. Here we have $\mu V_{E}-\nu V_{I}$ $=2.76 \mathrm{mVms}^{-1}$ and the asymptotic level of the membrane potential given by $(3.8)$ is $E(X(+\infty))=$ $13.80 \mathrm{mV}$. Taking again $\sigma^{2}=0.03$, then $\operatorname{Var}(X(+\infty))=143.12 \mathrm{mV}^{2}$ and the parameters of the 
distribution (3.12) are, $A=10.45$ and $B=2.88$ which induces that $x_{m}=8.29 \mathrm{mV}$.

The moments of the FPT for Stein's model with reversal potentials are: mean $=5.83 \mathrm{~ms}, \mathrm{CV}=$ 0.53. Using (5.2) with neglected second term in the parenthesis we get the mean approximated by 2.10 ms. For $\sigma^{2}=0.03,(5.2)$ gives the approximation $3.08 \mathrm{~ms}$. Now the asymptotic membrane potential is above the threshold and thus the approximation (5.6) gives $t^{*}=6.45 \mathrm{~ms}$ and (5.7) yields $\tilde{t}=5.68 \mathrm{~ms}$. From (4.5) we obtain $E\left(T_{10,0}\right)=3.73 \mathrm{~ms}$ and from (4.8) we can compute $C V=0.94$. Again, decreasing $\sigma^{2}$ the mean ISI becomes longer but in entirely different way than for Example 1 (Fig. 2a.). It is almost linear with finite limit as $\sigma$ tends to zero. Of course, the Ornstein-Uhlenbeck model has also the finite limit of the FPT for $\sigma$ equals to zero under the condition that the absolute term in the drift is positive. Also the dependency of $\mathrm{CV}$ on $\sigma$ has a different shape than in Example 1. The means of FPT of Stein's model with reversal potential and the model $(2.10)$ practically coincides for $\sigma^{2}=0.0015 ; \mathrm{E}\left(T_{10,0}\right)=$ $5.82 \mathrm{~ms}$ and $\mathrm{CV}=0.38$.

Dependency of the mean ISI (firing rate) on the input intensity is one of the most common characteristics of neural model as well as experimental data. We can see on Fig. 4 how, for different $\sigma$, this dependency looks for the present model.

The Feller model.

The membrane potential can fluctuate only in the interval $\left(V_{I}, S\right)$ if the firing at the threshold is assumed. It means, taking into account the parameters in the above examples, that for both the models (2.4) and its diffusion counterpart (2.10), the nonlinearity caused by the excitatory reversal potential is not substantial. The direct consequence would be similarity between the model (2.10) and (2.11). They differ only in the infinitesimal variances and this difference is maximally about $5 \%$ as it is illustrated on Fig. 5. ( $\sigma$ for (2:11) was taken in that way that the infinitesimal variances coincide at the resting level). Wilbur and Rinzel (1983) used $V_{E}=70 \mathrm{mV}$, which is lower than the value used in the above presented exmples, but the difference between the two models does not become much bigger (see Fig. 5). Balossino et al. (1992) used in the comparison of the Feller model with the Ornstein-Uhlenbeck a set of parameters close to ours $\left(V_{I}=-10 \mathrm{mV}, S=10 \mathrm{mV}, \tau=5 \mathrm{~ms}, x_{0}=0 \mathrm{mV}, \mu=\nu=0\right)$ except for the higher value of $\sigma$. Their analysis concentrated on the computation of density function and on the 
demonstration of the asymptotic properties (Poissionian trend) and thus it can serve only for a crude comparison with the simulated histograms in Fig. 3. Generally, one can expect the same fit of the two models (2.10) and (2.11) with experimental data and the preference should follow only from the biological reasoning during the models construction.

\section{Perspectives and Conclusions}

After spike generation is a relatively short period of different behaviour which is neglected throughout this paper. These features as refractoriness (Clay and Goel, 1973) or afterhyperpolarization (Lánský et al., in press) are ignored in this paper, however, they can be introduced into the model in the same way as it is described in the cited papers. Also the spatial extent of the neuron reflected either by variable initial depolarization $x_{0}$ (Lánský and Smith, 1988) or by segmentation of the model neuron at least into two parts (Kohn, 1989; Rospars and Lánský, submitted) can be considered using the introduced model. Time-dependent infinitesimal moments, reflecting a non-constant neuronal input, can be introduced into the diffusion models with reversal potentials in the same way as into the Ornstein-Uhlenbeck model (Lánský, 1984). The models with the time dependent terms can describe the presence of burst-type activity (Ricciardi, 1982), the existence of adaptation phenomena, the phase-locking effect (Lánský et al., 1992). However, to solve the FPT problems for these models can be done only by simulation or by using the numerical techniques. Generally, the model studied in this paper can replace the existing diffusion neuronal models in all their applications.

The Ornstein-Uhlenbeck process has been considered as an appropriate neuronal model for at least two decades. The models with non-constant infinitesimal variance reflect in a better way the features of real neurones and thus, taking this as the criterion, there is no doubt about their superiority over the Ornstein-Uhlenbeck model. Usually, introducing more detailed description of reality into a model makes this model more difficult for mathematical treatment, but this is not the case as we are able to find the results on the approximately same level as for the Ornstein-Uhlenbeck model. Let us compare this situation with the difference between the Wiener process with drift as neuronal model and the Ornstein-Uhlenbeck model. There, for the former one the FPT density can be analytically computed which allows comparison of the model with experimental data while for the latter the methods for model 
verification are substantially more complicated (Lánský, 1983) and thus have never been used. So, the Ornstein-Uhlenbeck model has been considered as better from the theoretical point of view, however, its experimental verification has been always performed only at the qualitative level. On the other hand, so often found fit of the Wiener model to the experimental data does not prove that the model is correct, for disccusion see Levine (1992). Such coincidence between the data and an oversimplified model may cause that the estimated physiological parameters as well as the input parameters are confused by the neglected properties of the neuron, (the spontaneous decay of the membrane potential comparing these two models). So we can conclude that the models with non-constant infinitesimal variances are more realistic and suitable for the description of real neurons than the Ornstein-Uhlenbeck model. The open question which remains to solve is which of these model is the most appropriate one.

ACKNOWLEDGEMENT: Supported in part by Office of Naval Research Grant N00014-90-J-1646-1, NAS-CSAS scientist exchange program and Czechoslovak Academy of Sciences grant \#71143.

\section{REFERENCES}

ABRAMOWITZ, M. \& STEGUN I.A. (Eds) (1964) Handbook of mathematical functions. New York: Dover.

BALOSSINO, N., BUONOCORE, A. \& SACERDOTE, L. (1992) On two neuronal diffusion models, In: Cybernetics and System Research'92 (Trappl, R. ed). Singapore: World Scientific.

CAPOCELLI, R.M. \& RICCIARDI, L.M. (1971) Diffusion approximation and the first passage time for a model neuron, Kybernetik 8, 214-223.

CLAY, J.R. \& GOEL, N.S., (1973), Diffusion models for firing of a neuron with varying threshold. $J$. theor. Biol. 39, 633-644.

DURBIN, J. (1971) Boundary-crossing probabilities for the Brownian motion and Poisson processes and techniques for computing the power of the Kolmogorov-Smirnov test, J. Appl. Probab. 8, 431-453.

GIORNO, V., LÁNSKÝ, P., NOBILE, A.G. \& RICCIARDI, L.M. (1988) Diffusion approximation and first-passage-time problem for a model neuron. III. A birth-and-death process approach. Biol. Cybern. $58,387-404$. 
GIORNO, V., NOBILE, A.G., RICCIARDI, L.M. \& SATO, S. (1989) On the evaluation of first-passagetime probability densities via nonsingular integral equations. Adv. Appl. Probab. 21, 20-36.

GOEL, N.S. \& RICHTER-DYN, N. (1974) Stochastic models in biology. New York: Academic Press.

HANSON, F.B. \& TUCKWELL, H.C. (1983) Diffusion approximation for neuronal activity including synaptic reversal potentials. J. Theor. Neurobiol. 2, 127-153.

KALLIANPUR, G. (1983) On the diffusion approximation to a discontinuous model for a single neuron, In: Contributions to Statistics (Sen, P.K. ed). Amsterdam: North-Holland.

KALLIANPUR, G. \& WOLPERT, R.L. (1987) Weak convergence of stochastic neuronal models, In: Stochastic methods in biology. Berlin: Springer.

KARLIN, S. \& TAYLOR, H.M. (1975) A first course in stochastic processes. New York: Academic Press.

KEILSON, J. \& ROSS, H.F. (1975) Passage time distributions for Gaussian Markov (OrnsteinUhlenbeck) statistical processes. Selected Tables in Mathematical Statistics 3, 233-327.

KLOEDEN, P.E. \& PLATEN, E. (to appear) The numerical solution of stochastic differential equations. Berlin: Springer.

LÁNSKÁ, V. (1988) On the diffusion approximation of the generalized Stein's neuronal model with synaptic reversal potentials, In: Transactions of the Tenth Prague Conference on Information Theory, Statistical Decision Functions, Random Processes (Víšek, J.A. ed). Prague: Academia.

LANSKY, P. (1983) Inference for the diffusion models of neuronal activity. Math. Biosci. 67, 247-260.

LÁNSKY, P. (1984) On approximations of Stein's neuronal model. J. theor. Biol. 107, 631-647.

LÁNSKY, P. \& LÁNSKA, V. (1987) Diffusion approximations of the neuronal model with synaptic reversal potentials. Biol. Cybern. 56, 19-26.

LANSKÝ, P. \& MUSILA, M. (1991) Variable initial depolarization in the Stein's neuronal model with synaptic reversal potentials. Biol. Cybern. 64, 285-291.

LANSKY, P., MUSILA, M. \& SMITH, C.E. (in press) Effects of afterhyperpolarization on neuronal firing. BioSystems.

LANSKÝ, P., ROSPARS, J.P. \& VAILLANT, J. (1992) Some neuronal models with oscillatory input, In: Cybernetics and System Research '92 (Trappl, R. ed). Singapore: World Scientific. 
LANSKY, P. \& SMITH, C.E. (1989) The effect of random initial value in neuronal first-passage-time models. Math. Biosci. 93, 191-215.

LEVINE, M.W. (1991) The distribution of intervals between neural impulses in the maintained discharges of retinal ganglion cells. Biol. Cybern. 65, 459-467.

MUSILA, M. \& LANSKY P. (1991) Generalized Stein's model for anatomically complex neurons. BioSystems 25, 179-191.

MUSILA, M. \& LÁNSKYY P. (1992) A neuronal model with variable synaptic input effect. Cyb. Sys. 23, 29-40.

MUSILA, M. \& LANSKY P. (in press) Simulation of a diffusion process with randomly distributed jumps in neuronal context. Int. J. Biomed. Comput.

NOBILE, A.G., RICCIARDI, L.M. \& SACERDOTE, L. (1985) Exponential trends of first-passage-time densities for a class of diffusion processes with steady-state distribution. J. Appl. Probab. 22, 611-618.

PACUT, A. \& DABROWSKI, L. (1988) Delayed-exponential approximation of a linear homogeneous diffusion model of neuron. Biol. Cybern. 59, 395-404.

PARDOUX, E. \& TALAY, D. (1985) Discretization and simulation of stochastic differential equations. Acta Appl. Math. 3, 23-47.

RICCIARDI, L.M. (1982) Diffusion approximations and computational problems for single neurons activity, In: Competition and cooperation in neural networks (Amari, S. \& Arbib, M.A. eds). New York: Springer.

RICCIARDI, L.M. \& SACERDOTE, L. (1979) The Ornstein-Uhlenbeck process as a model for neuronal activity. Biol. Cybern. 35, 1-9.

RICCIARDI, L.M., SACERDOTE, L. \& SATO, S. (1983) Diffusion approximation and first-passagetime problem for a model neuron. Il. Outline of a computation method. Math. Biosci. 64, 29-44.

RICCIARDI, L.M. \& SATO, S. (1990) Diffusion process and first-passage-times problems, In: Lectures in applied mathematics and informatics (Ricciardi, L.M. ed). Manchester: Manchester Univ. Press.

ROSPARS, J.P. \& LANSKY P. (submitted) Stochastic model neuron without resetting of dendritic potential. Application to first and second order neurons in olfactory system.

RÜMELIN, W. (1982) Numerical treatment of stochastic differential equations. SIAM J. Numer. Anal. 
3, 604-613.

SATO, S. (1978) On the moments of the firing interval of diffusion approximated neuron. Math. Biosci. 34, 53-70.

SCHMIDT, R.F. ed. (1984) Fundamentals of neurophysiology, 2nd edition. Berlin: Springer.

SMITH, C.E. (1992) A note on neuronal firing and input variability. J. theor. Biol. 154, 271-275

SMITH, C.E. \& SMITH, M.V. (1984) Moments of voltage trajectories for Stein's model with synaptic reversal potentials, J. Theor. Neurobiol. 3, 67-77.

STEIN, R.B. (1965) A theoretical analysis of neuronal variability. Biophys. J. 5, 173-195.

STEIN, R.B. (1967) Some models of neuronal variability. Biophys. J. 7, 37-67.

TSURUI, A. \& OSAKI, S. (1976) On a first-passage problem for a cumulative process with exponential decay. Stoch. Proc. Appl. 4, 79-88.

TUCKWELL, H.C. (1979) Synaptic transmission in a model for stochastic neural activity. J. theor. Biol. 77, 65-81.

TUCKWELL, H.C. (1988) Introduction to theoretical neurobiology, Vol. 2: Nonlinear and stochastic theories. Cambridge: Cambridge Univ. Press.

TUCKWELL, H.C. \& COPE, D.K. (1980) The accuracy of neuronal interspike times calculated from a diffusion approximation. J. theor. Biol. 83, 377-387.

WAN, F.Y.M. \& TUCKWELL, H.C. (1982) Neuronal firing and input variability. J. Theor. Neurobiol. $1,197-218$.

WILBUR, A.J. \& RINZEL, J. (1983) A theoretical basis for large coefficient of variation and bimodality in neuronal interspike interval distribution. J. theor. Biol. 105, 345-368.

VASUdEVAN, R. \& VITTAL, P.R. (1982) Time-dependent barriers and first-passage times - Different types of neuronal models. Neurol. Res. 4, 63-87.

\section{APPENDIX A}

According to Abramowitz and Stegun (1965) the Kummer's function can be expressed in the integral form as

$F$ is a hypergeometric function

$$
M(a, b, s)=\frac{1}{B(b-a, a)} \int_{0}^{1} \mathrm{e}^{s t} t^{a-1}(1-t)^{b-a-1} \mathrm{~d} t
$$




$$
F(a, b, c ; x)=1+\frac{a b}{1 ! c} x+\frac{a(a+1) b(b+1)}{2 ! c(c+1)} x^{2}+\cdots
$$

and the explicit expressions for the Jacobi polynomials $P_{i}^{\epsilon, b}(y)$ are

$$
P_{i}^{\epsilon, b}(y)=\frac{1}{2^{i}} \sum_{m=0}^{i}\left(\begin{array}{c}
i+c \\
m
\end{array}\right)\left(\begin{array}{c}
i+b \\
i-m
\end{array}\right)(y-1)^{i-m}(y+1)^{m}
$$

For the interrelations between hypergeometric function, Jacobi polynomials, Kummer's, Beta and Gamma functions see Abramowitz and Stegun (1965).

\section{APPENDIX B}

The constant $C_{2}$ is determined by comparing the mean FPT computed directly from the recursive relation (1.12) for the moments of the FPT with that obtained from the Laplace tranformation of the FPT density using (1.11). For the first moment $M_{1}(S \mid y)=\mathrm{E}\left(T_{S, y}\right)$ we have

$$
\frac{1}{2} \sigma^{2} y(1-y) \frac{\partial^{2} M_{1}(S \mid y)}{\partial y^{2}}+(-\alpha y+\beta) \frac{\partial M_{1}(S \mid y)}{\partial y}+1=0
$$

with the initial condition $M_{1}(S \mid S)=0$. The second initial condition is derived using the following argument. For $Y(t)=0$ the drift coefficient is $\beta$ and diffusion coefficient equals to 0 . Conditionally on $Y(t)=0, \quad Y(t+\Delta)=\beta \Delta,(\Delta>0$ and small $)$. Then

$$
M_{1}(S \mid 0)=\Delta+\mathrm{E}\left[M_{1}(S \mid 0) \mid Y(t)=0\right]=\Delta+M_{1}(S \mid \Delta \beta)+o(\Delta)
$$

Therefore $\partial M_{1}(S \mid 0) / \partial y=-1 / \beta$. Now, the solution of the above mentioned differential equation is unique and it reads

$$
M_{1}(S \mid y)=\frac{2}{\sigma^{2}} \int_{y}^{S} s(x) \int^{x} \frac{1}{s(z) z(1-z)} \mathrm{d} z
$$

where $s(x)=x^{-\frac{2 \beta}{\sigma^{2}}(1-x) \frac{2(\beta-\alpha)}{\sigma^{2}}}$. The inner integral is expressed by infinite sum (Abramowitz and Stegun, 1965) and then after integration we get

$$
M_{1}\left(S \mid x_{0}\right)=\frac{S-y}{\beta}+\sum_{n=0}^{\infty} \frac{B\left(\frac{2 \beta}{\sigma^{2}}+1, n+1\right)}{B\left(\frac{2 \alpha}{\sigma^{2}}, n+1\right)} \frac{S^{n+2}-y^{n+2}}{\beta(n+2)}
$$

which is the same as (4.5) which is derived from (4.2) under the condition $C_{2}=0$. 


\section{Text to figures}

Fig. 1. Simulated histograms of the membrane potential in Example 1 for $t=3 \mathrm{~ms}$ and $t=10 \mathrm{~ms}$. The theoretical stationary density (3.12) is superimposed for both. In the first case $(t=3 \mathrm{~ms})$ there is a lack of fit (tested by $\chi^{2}$ test at $5 \%$ level; $\chi_{17}^{2}=24.53$ ) caused mainly by lower number of the membrane potential values above $10 \mathrm{mV}$. For $t=10 \mathrm{~ms}$ the fit has not been rejected. Even for $t=5 \mathrm{~ms}$ (histogram is not presented) the fit has not been rejected and thus time longer than $5 \mathrm{~ms}$ seems to be sufficient to bring the membrane potential to its stationary distribution.

Fig. 2a. Dependency of the mean ISI on the parameter $\sigma$ as given by equation (4.5) for Example 1 denoted by crosses and for Example 2 denoted by circles. The boundary in $\sigma$ above which the inhibitory reversal potentials starts to be regular is pesented. For large $\sigma$ the model outputs become simmilar while for low $\sigma$ the behavior is qualitatively different

Fig. 2b. Dependency of the coefficient of variation CV of the ISIs on the parameter $\sigma$ as given by equation (4.5) and (4.8) for Example 1 denoted by crosses and for Example 2 denoted by circles. Again as for the means, CVs coincide in both examples for large $\sigma$ and behave differently for low $\sigma$.

Fig. 3a. Simulated histogram of ISIs in Example 1 for $\sigma^{2}=0.03$ together with exponential density having the mean equal to the theoretical mean as it follows from equation (4.5). The fit has not been rejected (tested by $\chi^{2}$ test at $5 \%$ level).

Fig. 3b. Simulated histogram of ISIs in Example 1 for $\sigma^{2}=0.0063$. In contrast to the Fig. 3a. the mode is not in the first bin.

Fig. 4. Dependency of the mean ISIs in Example 2 on the excitation parameter $\mu$ for different values of the parameter $\sigma$. From upper line in the order $\sigma^{2}=0.001, \sigma^{2}=0.005, \sigma^{2}=0.01, \sigma^{2}=0.02, \sigma^{2}=0.03$,

Fig. 5. The dependency of the infinitesimal variance $\sigma \sqrt{\left(V_{E}-X\right)\left(X-V_{I}\right)}$ on the actual value of the membrane potential $X$ for the model (2.10) with the parameter $V_{E}=100 \mathrm{~ms}$ denoted by - - and denoted by - - when the parameter $V_{E}=70 \mathrm{~ms}$ was applied. The values of the infinitesimal variances are scaled by $\sigma=10$ for better appearance. At the lower part of the figure is presented the percentage difference of this coefficient to the corresponding coefficient for the model (2.11). The infinitesimal variance for $(2.11)$ is scaled in such a way that it equals to that for $(2.10)$ at $x=0$, so we present

$$
100 \frac{10 \sqrt{V_{E}} \sqrt{X-V_{I}}-10 \sqrt{\left(V_{E}-X\right)\left(X-V_{I}\right)}}{10 \sqrt{\left(V_{E}-X\right)\left(X-V_{I}\right)}}=100 \sqrt{V_{E}} / \sqrt{V_{E}-X}-100 .
$$

It can be seen that even for lower excitatory reversal potential the percentage difference is far below $10 \%$. 


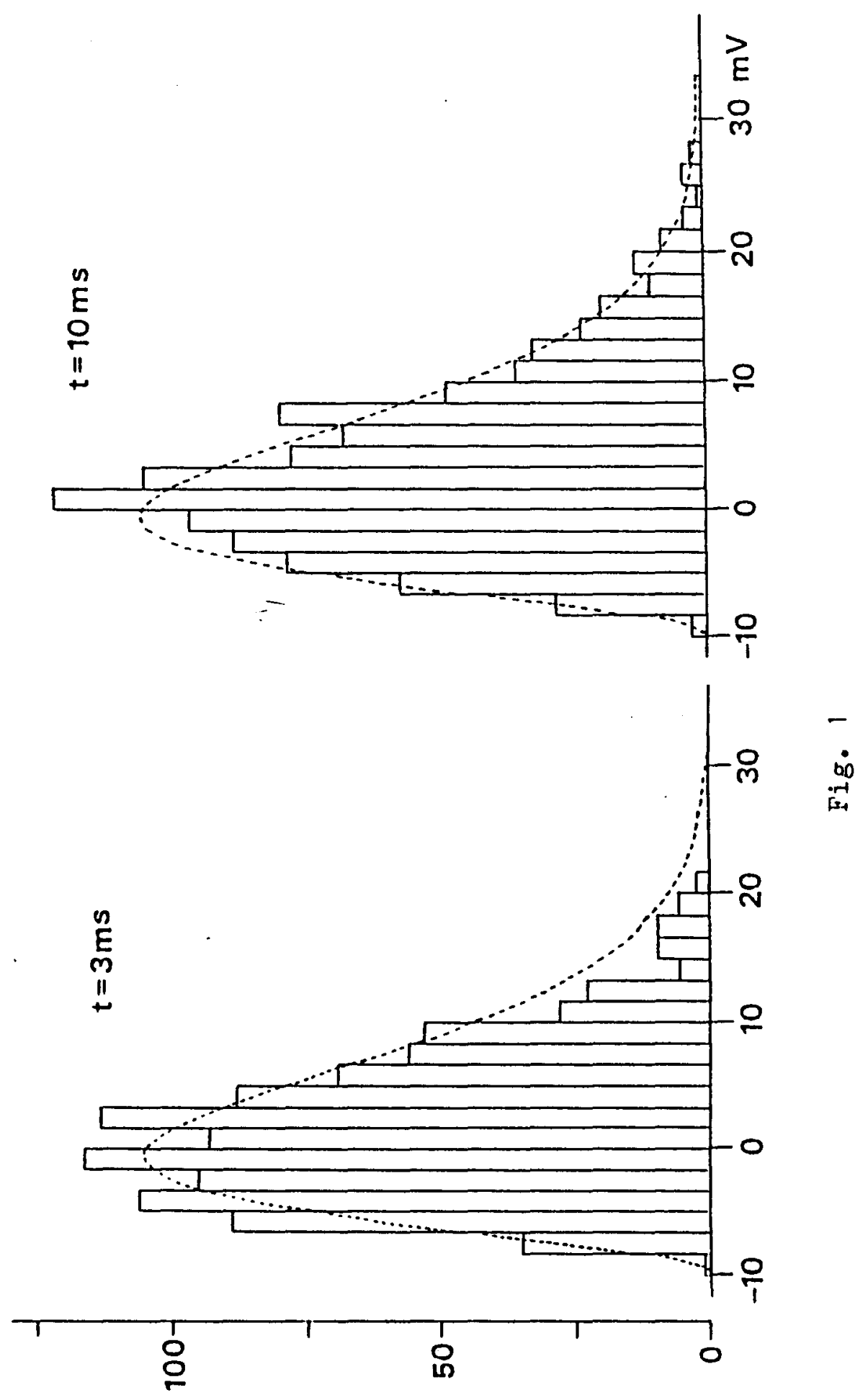




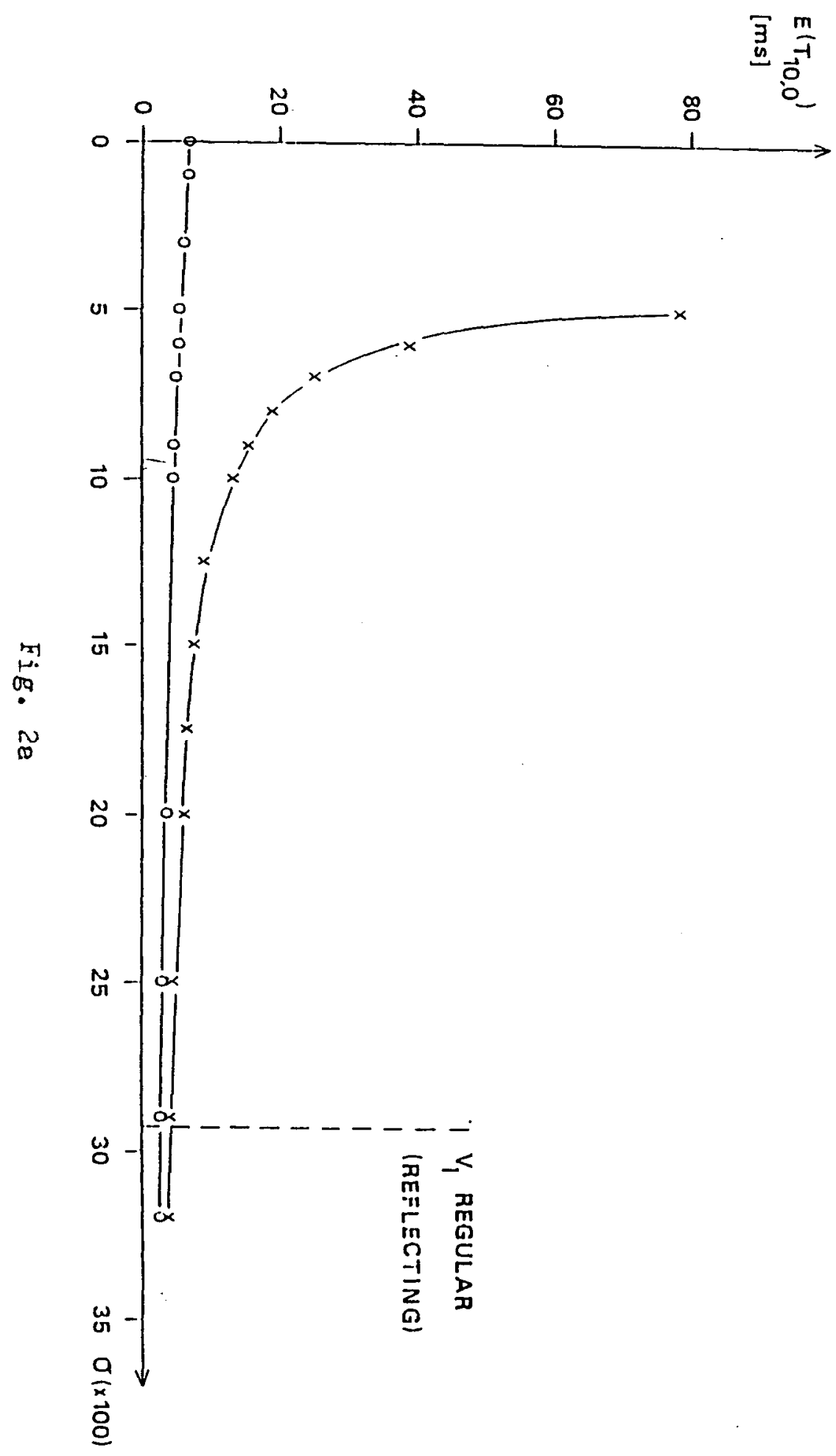




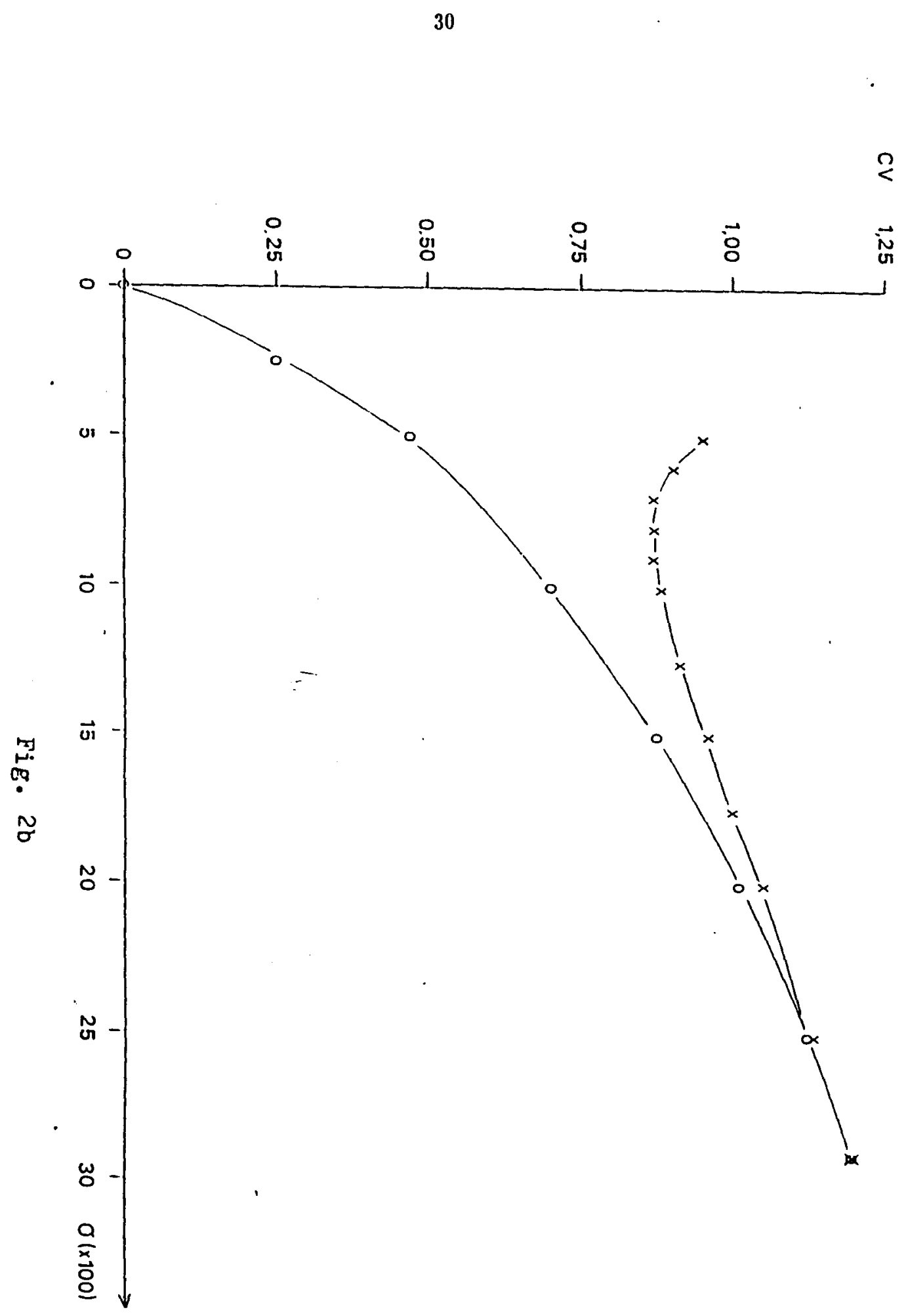



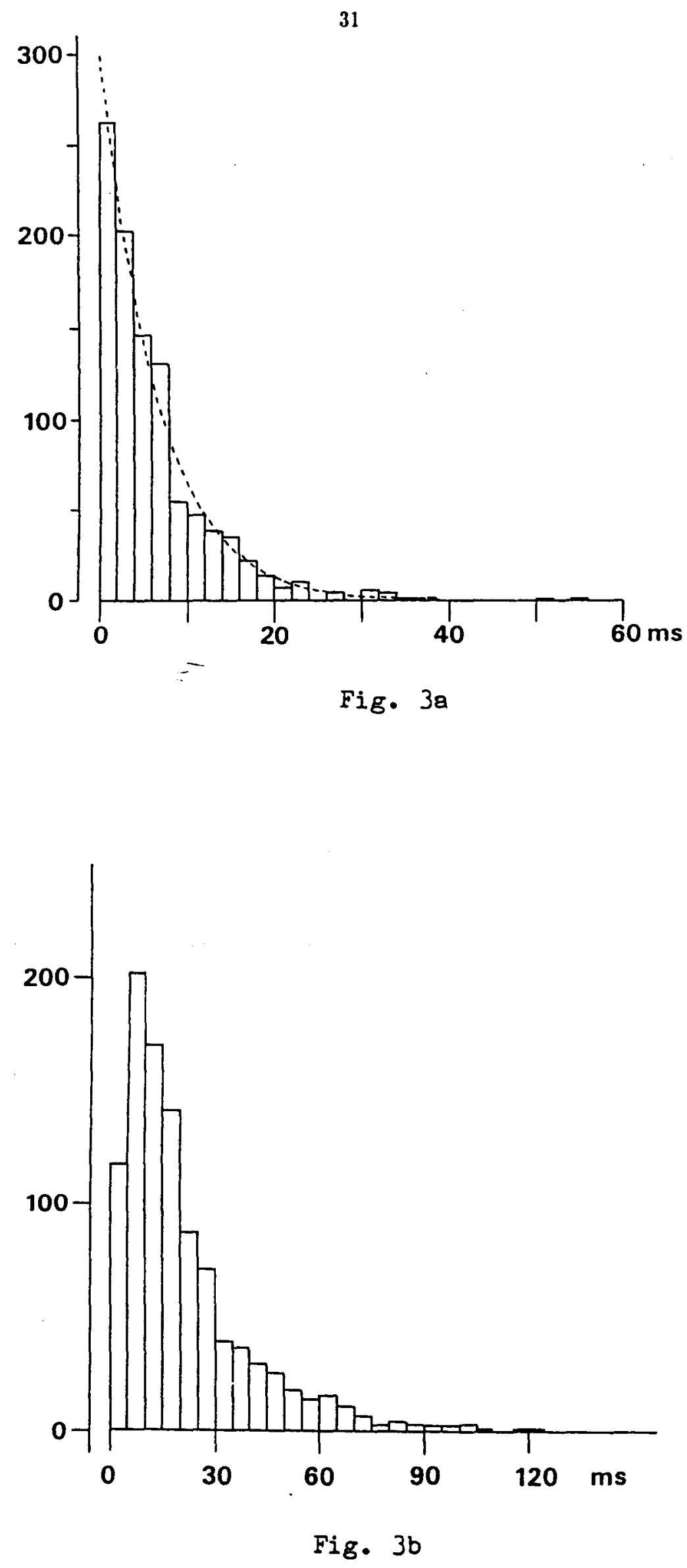


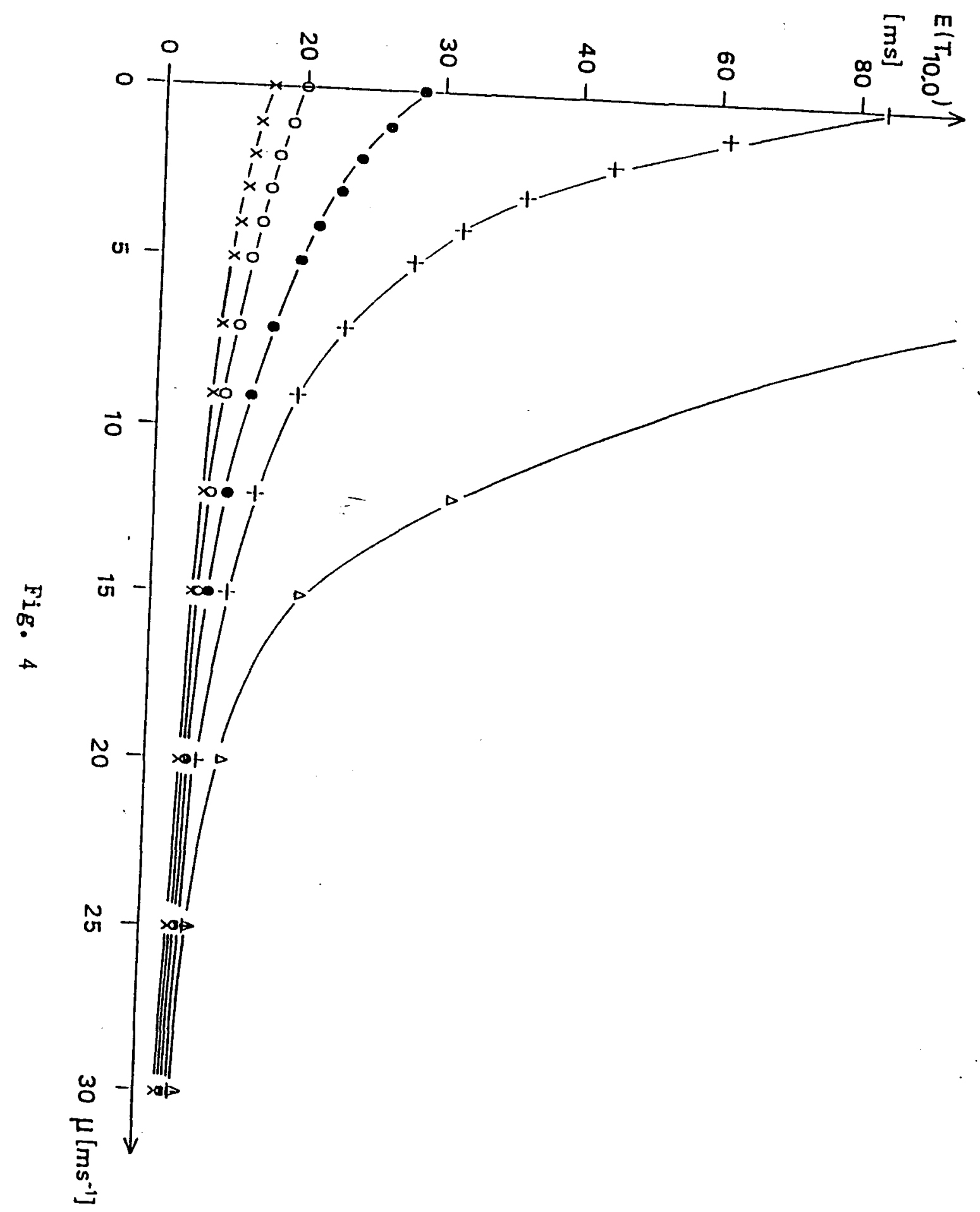




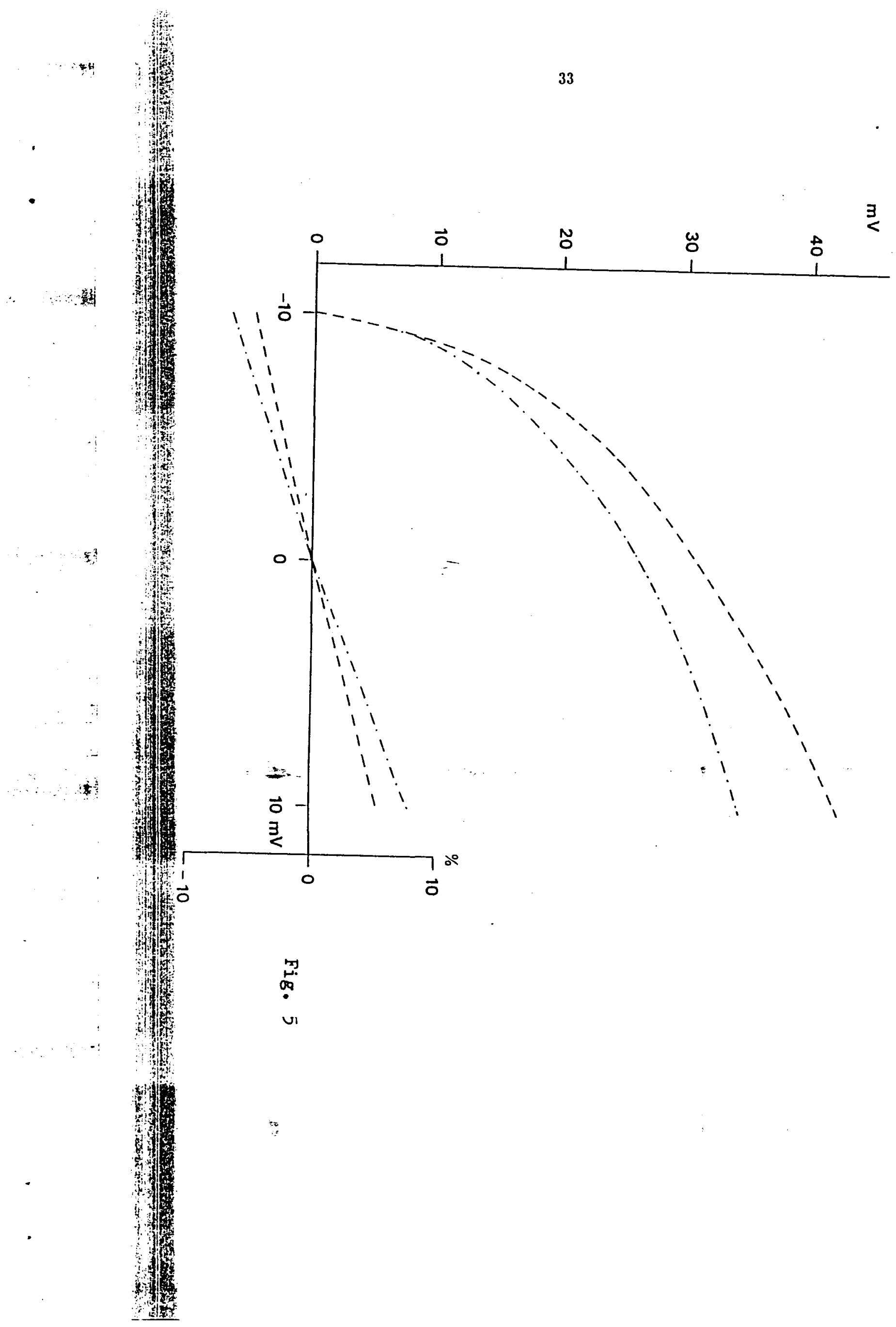


- 1

\section{REPORT DOCUMENTATION PAGE}

\begin{tabular}{l} 
19. REPORT SECURITY CLASSITICATION \\
UNCLASSIFIED \\
\hline 20. SECUKIIY CLASSIFICAIION AUTHORITY \\
2b. DEGLASSIFICATIONI DOWNGRADING SCHEOULE
\end{tabular}

Ib. RESTRICTIVE MARKINGS

4. PERFORMING ORGANIZAIION REPORI NUMBER(S)

3. DISTRIBUTIONIAVAILABILIIY OF REPORT

Approved for public Release:

Distribution Unlimited

5. MONIIOIING ORGANIZAIION REPORI NUMHER(S)

6a. NAME OF PERFORMING ORGANIZAIION

North Caroliua state Univ.

be Aldinfiss (Cify, Stale, and zip rodu)

Dept of Statistics

Raleigh, North Caroliua 27695-8203

8. NAME OF FUNDING/SPONSCAING ORGANIZATION

Office of Naval Research

Bb. OIFICE SYMBOL (If spolicsble)

ONR

86. AODRESS (Ciry, Sidte, ond 2IP Code)

Repartment of the Navy

8Qu North Quincy Street

Arlington, Virginia 22217-5000

11. IITLE (include Securioy Clasufication)

Synaptic Transmission in a Diffusion Model for Neural Activity (Unclassified)
7.. NAME OF MONITORING ORGANIZAIION

Office of Naval Research

Department of the Navy

In Aliliks ss (cily. Itole, aind Jir Conde)

800 North Quincy Street

Arlington, Virginia -. 22217-5000

9. PROCUREMINI INSIRUMENT IDENIIFICATION NUMBER

N00014-90-J-1646-1

10 SOUACE OF FUNDING NUMBERS

\begin{tabular}{l|l|l|l}
\hline $\begin{array}{l}\text { PHOGRAM } \\
\text { ELEMENT NO. }\end{array}$ & $\begin{array}{l}\text { PROJECT } \\
\text { NO. }\end{array}$ & $\begin{array}{l}\text { TASK } \\
\text { NO. }\end{array}$ & $\begin{array}{l}\text { WORK UNIT } \\
\text { ACCESSION NO. }\end{array}$ \\
\hline
\end{tabular}

12. PERSONAL AUTHOR(S) Vera Lanska, Petr Lansky, Charles E. Smith

\begin{tabular}{l|l} 
13. TYPE OF REPCRT & 1?b TIM.AE COVEREO
\end{tabular} TECHNICAL

16. SUPPLEMENTARY NOTATION

\begin{tabular}{|c|c|c|}
\hline 17. & \multicolumn{2}{|c|}{ COSATI COOES } \\
\hline FIELO & GROUP & SUB-GROUP \\
\hline & & \\
\hline & & \\
\hline
\end{tabular}

48. SUBJECT TERMS (Continue on reverse if necessary and identify by block number)

19. ABSTRACT (Continue on reverse It necessary and identify by bloxk number)

Equations for a diffusion neuronal model describing the production of nerve impulses

have been derived for the case in which the noisy depolarizations of the membrane potential

are restricted by the reversal potentials. Identifying neuron firing intervals with the

first-passage time distribution for the associated process allows us to compute the inter-

spike interval statistics and relate them to the parameters characterizing the neuronal

input. Methods for approximation of the first two moments of the interspike intervals are

proposed. The analytical results are numerically illustrated and simultaneously the

computer simulations were performed for. the same purpose. A comparison of the achieved

results is made with those from other diffusion models or the models with discontinuous

trajectories. The model is proposed mainly as an alternative to the Ornstein-Uhlenbeck

neuronal model.

20. DISTAIBUTION /AVALAABLITY OF ABSIRACT

[Q] UHCLASSIFIFDNNLIMIICo $\mathbb{Q}$ Same AS RPT.

2. NAME OF RESPONSIDLE INDIVIOUAL

DORIC USEAS

21. ABSIRACT SECURITY CLASSIFICATION

UNCLASSIFIED

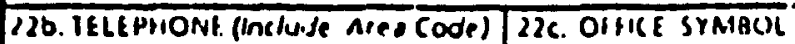

